\title{
SYNTHESIS, CHARACTERIZATION AND ANTITUMOUR ACTIVITIES OF DI-n-BUTYL- AND DIMETHYLTIN D-(+)-CAMPHORATES
}

\author{
Marcel Gielen*1a, Hassan Dalil1a, Bernard Mahieu², \\ Dick de Vos ${ }^{3}$, Monique Biesemans ${ }^{1}$, and Rudolph Willem ${ }^{1}$ \\ 1 Vrije Universiteit Brussel, Pleinlaan 2, B-1050 Brussels, Belgium \\ a Department of General and Organic Chemistry, Faculty of Applied Sciences \\ b High Resolution NMR Centre \\ 2 Catholic University of Louvain, CPMC, B-1348 Louvain-la-Neuve, Belgium \\ 3 Medical Department, Pharmachemie B. V., NL-2003 RN Haarlem, the Netherlands
}

\begin{abstract}
The synthesis and characterization di-n-butyl- and dimethyltin $D-(+)$-camphorates, respectively compounds 1 and 2 , are reported. Compound 1 displays antitumoural activity in vitro, and is more active than cisplatin, 5 -fluorouracil and etoposide against seven tumoural cell lines of human origin, but less active than methotrexate and doxorubucin.
\end{abstract}

Several diorganotin derivatives of dicarboxylic acids are active in vitro against human tumoural cell lines ${ }^{1-6}$. Nevertheless, diorganotin derivatives of dicarboxylic acids with proven antitumour activity in vitro remain rather rare. The present paper reports the synthesis and characterization of two novel diorganotin dicarboxylates, di-n-butyl- and dimethyltin D-(+)-camphorates, in order to further investigate the influence of the structure of such a dicarboxylate moiety on antitumour properties ${ }^{7}$. The antitumour activity of the di-n-butyltin compound is presented, that of the dimethyltin compound being provided for comparison.

Di-n-butyl- and dimethyltin D-(+)-camphorates, respectively compounds 1 and 2 , were prepared by condensing the appropriate diorganotin oxide with camphoric acid in refluxing toluene/ethanol 4/1 under elimination of the azeotrope water/toluene/ethanol8-10. They were recrystallized from ethanol/petroleum ether, m.p. $138-140^{\circ} \mathrm{C}$, yield: $90 \%$, and methylene chloride/hexane, m.p. 258$259{ }^{\circ} \mathrm{C}$, yield: $96 \%$, respectively. The Mössbauer parameters of compounds 1 and 2 are respectively I.S.: 1.39 , Q.S.: $3.32, \Gamma_{1}: 1.06, \Gamma_{2}: 0.92$ and I.S.: 1.35, Q.S.: $3.89, \Gamma_{1}: 0.93, \Gamma_{2}: 1.02$ $\mathrm{mm} / \mathrm{s} .{ }^{1} \mathrm{H}$ and ${ }^{13} \mathrm{C}$ NMR data are presented in Table 1.

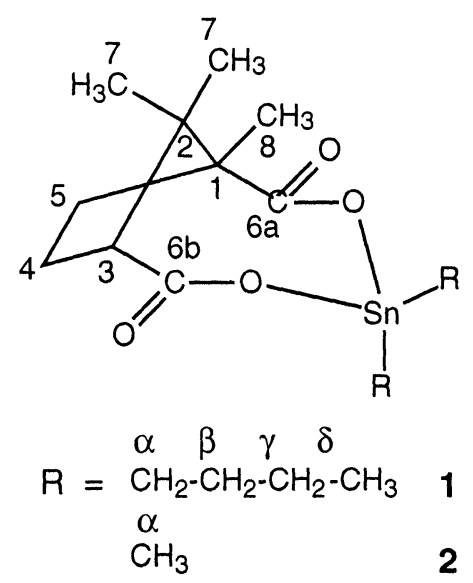

The molecular weight of 2 was determined by cryoscopy in camphor (calc. for $\mathrm{C}_{12} \mathrm{H}_{20} \mathrm{O}_{4} \mathrm{Sn}$ : 347; found: $343 \pm 52$ Dalton. 


\begin{tabular}{|c|c|c|c|c|c|}
\hline & \multicolumn{3}{|c|}{${ }^{1} \mathrm{H}$} & \multicolumn{2}{|c|}{${ }^{13} \mathrm{C}$} \\
\hline & 1 & 2 & & 1 & 2 \\
\hline & & & $\begin{array}{l}\mathrm{C}_{1} \\
\mathrm{C}_{2}\end{array}$ & $\begin{array}{l}56.2 \\
46.7\end{array}$ & $\begin{array}{l}56.0 \\
46.9\end{array}$ \\
\hline $\mathrm{H}_{3}$ & $\mathrm{t}: 2.86$ [9] & t: 2.85 [9] & $\mathrm{C}_{3}$ & 52.6 & 52.5 \\
\hline $\begin{array}{l}\mathrm{H}_{4 a} \\
\mathrm{H}_{4 b}\end{array}$ & $\begin{array}{l}\mathrm{m}: 2.22-2.10 \\
\mathrm{~m}: 1.90-1.77\end{array}$ & $\begin{array}{l}\mathrm{m}: 2.22-2.10 \\
\mathrm{~m}: 1.89-1.76\end{array}$ & $\mathrm{C}_{4}$ & 23.2 & 22.9 \\
\hline $\begin{array}{l}H_{5 a} \\
H_{5 b}\end{array}$ & $\begin{array}{c}\text { ddd: } 2.53[12,8,8] \\
\text { m: } 1.56-1.47\end{array}$ & $\begin{array}{c}\text { ddd: } 2.53[12,8,8] \\
\text { m: } 1.55-1.45\end{array}$ & $\mathrm{C}_{5}$ & 32.9 & 32.7 \\
\hline & & & $\begin{array}{l}\mathrm{C}_{6 a} \\
\mathrm{C}_{6 b}\end{array}$ & $\begin{array}{l}186.4 \\
184.2\end{array}$ & $\begin{array}{l}186.3 \\
184.0\end{array}$ \\
\hline $\begin{array}{l}\mathrm{H}_{7 a} \\
\mathrm{H}_{7 \mathrm{~b}}\end{array}$ & $\begin{array}{l}\text { s: } 1.35 \\
\text { s: } 0.87\end{array}$ & $\begin{array}{l}\text { s: } 1.33 \\
\text { s: } 0.83\end{array}$ & $\begin{array}{l}\mathrm{C}_{7 \mathrm{a}} \\
\mathrm{C}_{7 \mathrm{~b}}\end{array}$ & $\begin{array}{l}22.7 \\
21.5\end{array}$ & $\begin{array}{l}22.8 \\
21.5\end{array}$ \\
\hline $\begin{array}{c}\mathrm{H}_{8} \\
\mathrm{H}_{\alpha-\beta}\end{array}$ & $\begin{array}{c}\mathrm{s}: 1.23 \\
\mathrm{~m}: 1.66-1.51\end{array}$ & $\begin{array}{c}\text { s: } 1.22 \\
\text { s: } 0.91(81)\end{array}$ & $\begin{array}{l}\mathrm{C}_{8} \\
\mathrm{C}_{\alpha} \\
\mathrm{C}_{\beta}\end{array}$ & $\begin{array}{c}22.4 \\
25.1(594 / 566) \\
26.8(35)\end{array}$ & $\begin{array}{c}21.9 \\
4.4(660 / 630) \\
-\end{array}$ \\
\hline $\mathrm{H}_{\gamma}$ & $\mathrm{m}: 1.48-1.34$ & - & $C_{\gamma}$ & 26.4 (97) & - \\
\hline $\mathrm{H}_{\delta}$ & $\mathrm{t}: 0.87$ [7] & - & $\mathrm{C}_{\delta}^{\prime}$ & 13.5 & - \\
\hline
\end{tabular}

Table 1: ${ }^{1} \mathrm{H}$ and ${ }^{13} \mathrm{C}$ NMR data of compounds 1 and $2\left(\mathrm{CDCl}_{3}\right)$. Chemical shifts in ppm vs. TMS; ${ }^{\mathrm{n} J}\left({ }^{1} \mathrm{H},{ }^{1} \mathrm{H}\right)$ coupling constants (between brackets), ${ }^{2} \mathrm{~J}\left({ }^{1} \mathrm{H},{ }^{119} \mathrm{Sn}\right)$ and ${ }^{n} \mathrm{~J}\left({ }^{13} \mathrm{C},{ }^{119 / 117} \mathrm{Sn}\right)$ coupling constants in $\mathrm{Hz}$ (bold in parentheses); d: doublet; t: triplet; s: singlet; $\mathrm{m}$ : complex pattern.

The ${ }^{117} \mathrm{Sn}$ NMR chemical shifts $\delta\left({ }^{117} \mathrm{Sn}\right)\left(\mathrm{CDCl}_{3}\right)(\mathrm{ppm})$ of compounds 1 and 2 are respectively -150.7 and $-124.8 \mathrm{ppm}$. These values are in agreement with the structure proposed, the absence of concentration effect ( 30 and $100 \mathrm{mg} / 0.5 \mathrm{ml}$ ) excluding any monomer - oligomer equilibrium.

Compounds 1 and 2 were screened in vitro against seven tumoural cell lines of human origin: MCF7 and EVSA-T, two mammary tumours, WiDr, a colon carcinoma, IGROV, an ovarian cancer, M19 MEL, a melanoma, A498, a renal cancer, and $\mathrm{H} 226$, a non-small cell lung cancer, as water/ethanol $99 / 1$ solutions.

The results of the antitumoural tests are summarized in table 2 and compared with the inhibition doses $I_{50}$ obtained for some clinically used reference compounds 11,12 , cisplatin, 5-fluorouracil, etoposide, methotrexate and doxorubicin.

\begin{tabular}{crrrrrrr}
\hline Compounds & MCF-7 & EVSA-T & WiDr & IGROV & M19 MEL & A498 & H226 \\
\hline $\mathbf{1}$ & 49 & 28 & 100 & 45 & 66 & 49 & 178 \\
$\mathbf{2}$ & 1342 & 903 & 3504 & 1006 & 1111 & 1548 & 764 \\
Cisplatin & 699 & 422 & 967 & 169 & 558 & 2253 & 3269 \\
5-Fluorouracil & 750 & 475 & 225 & 297 & 442 & 143 & 340 \\
Etoposide & 2594 & 317 & 150 & 580 & 505 & 1314 & 3934 \\
Methotrexate & 18 & 5 & $<3$ & 7 & 23 & 37 & 2287 \\
Doxorubicin & 10 & 8 & 11 & 60 & 16 & 90 & 199 \\
\hline
\end{tabular}

Table 2. Inhibition doses $I D_{50}$ of compounds 1 and 2 and of some reference compounds ${ }^{11}$ against seven tumoural cell lines of human origin

Compound 1 is more active than cisplatin, 5 -fluorouracil and etoposide against all cell lines, but less active than methotrexate and doxorubucin. The dimethyltin compound $\mathbf{2}$ is inactive, as usually ${ }^{13}$.

\section{Acknowledgements}

We thank Mrs. I. Verbruggen for recording the NMR spectra. We are grateful to Mr. H. J. Kolker, Dr. J. Verweij, Prof. Dr. G. Stoter, Dr. J. H. M. Schellens, Laboratory of Experimental Chemotherapy and Pharmacology, Department of Medical Oncology, Rotterdam Cancer Institute, $\mathrm{NL}$ - $3008 \mathrm{AE}$, Rotterdam, The Netherlands, for performing the in vitro tests. This research was supported by the Belgian "Nationaal Fonds voor Wetenschappelijk Onderzoek" (N.F.W.O. grant 
nr G.0054.96, M. G.) and the Fund for Scientific Research Flanders (Belgium, grant nr G.0192.98, R. W., M. B.).

\section{References}

1. R. Willem, M. Biesemans, M. Bouâlam, A. Delmotte, A. El Khloufi, M. Gielen, Appl. Organomet. Chem., 7 (1993), 311.

2. M. Gielen, M. Bouâlam, A. Meriem, B. Mahieu, M. Biesemans, R. Willem, Heteroatom Chem., 3 (1992), 449.

3. M. Gielen, R. Willem, Anticancer Res., 12 (1992), 269.

4. M. Gielen, M. Acheddad, B. Mahieu, R. Willem, Main Group Met. Chem., 14 (1991), 73.

5. A. Meriem, R. Willem, J. Meunier-Piret, M. Gielen, Main Group Met. Chem., 12 (1989), 187.

6. M. Gielen, E. Joosen, T. Mancilla, K. Jurkschat, R. Willem, C. Roobol, J. Bernheim, G. Atassi, F. Huber, E. Hoffmann, H. Preut, B. Mahieu, Main Group Met. Chem., 10 (1987), 147.

7. M. Gielen, Coord. Chem. Rev., 151 (1996), 41.

8. M. Gielen, A. El Khloufi, M. Biesemans, B. Mahieu, R. Willem, Bull. Soc. Chim. Belg., 101 (1992), 243.

9. M. Bouâlam, R. Willem, M. Biesemans, M. Gielen, Appl. Organomet. Chem., 5 (1991), 497.

10. M. Bouâlam, R. Willem, M. Biesemans, B. Mahieu, J. Meunier-Piret, M. Gielen, Main Group Met. Chem., 14 (1991), 41.

11. P. Skehan, R. Storeng, D. Scudiero, A. Monks, J. McMahon, D. Vistica, J. T. Warren, H. Bokesch, S. Kenney, M. R. Boyd, J. Natl. Cancer Inst., 82 (1990), 1107.

12. Y. P. Kepers, G. J. Peters, J. Van Ark-Otte, B. Winograd, H. M. Pinedo, Eur. J. Cancer, 27 (1991), 897.

13. M. Gielen, P. Lelieveld, D. de Vos, R. Willem, "Metal Complexes in Cancer Chemotherapy", ed. B. K. Keppler, VCH, Weinheim, 1993, chapter 17, pp. 383 - 390.

Received: May 28, 1998 - Accepted: July 15, 1998 Received in revised camera-ready format: July 16, 1998 